\title{
Dialisi incrementale: una procedura differente dalla dialisi infrequente
}

\section{Piergiorgio Bolasco' and Stefano Murtas ${ }^{2}$}

\begin{abstract}
Incremental dialysis: a procedure different from infrequent dialysis

The aim of the review is to provide the ultimate distinction between incremental dialysis and what is referred to as infrequent dialysis. Infrequent dialysis is defined as any hemodialytic schedule that includes fewer than three hemodialysis sessions per week. Nonetheless, infrequent dialysis could be defined as a therapy that requires close clinical and nutritional monitoring, and it should also be accompanied by a slightly hypoproteic dietetic approach. Incremental dialysis (CDDP) is a well-defined therapeutic program requiring significant clinical monitoring; it begins in the outpatient clinic when patients shift to a hypoproteic diet $(0.6 \mathrm{~g} / \mathrm{kg}$ per day) with or without essential amino acids and a low-salt diet supplemented by one dialysis session per week. The incremental dialysis program is strictly tailored on the residual renal function (RRF) trend and it represents a time variable for a therapeutic "bridge" that must provide a favourable metabolic status together with good quality of life. Recent studies have demonstrated lower mortality rates compared with thrice-weekly dialysis and a neutral input/output balance of the phosphorus pool. Further studies are needed to confirm the safety and validity of this therapeutic choice.
\end{abstract}

\section{Keywords}

Incremental hemodialysis, urea nitrogen appearance, once-weekly hemodialysis, hypoproteic diet

\section{Introduzione}

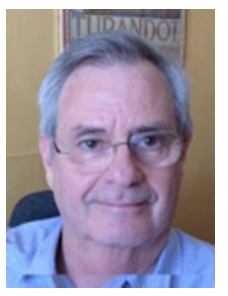

L'importanza di preservare la funzione renale residua (FRR) è stata per lungo tempo sottovalutata. Infatti la FRR ha dimostrato di essere una formidabile arma terapeutica che dovrebbe essere mantenuta il più a lungo possibile poiché anche un volume del filtrato glomerulare (VFG) $<3-5 \mathrm{~mL} / \mathrm{min}$ ha dimostrato potenzialità depurative significativamente superiori ai trattamenti emodialitici e peritoneali. ${ }^{1,2}$ Peraltro il modello di dialisi incrementale è di fatto stato mutuato dalla dialisi peritoneale $^{3,4}$ e successivamente traslato all'emodialisi. Il rene in fase terminale, pur perdendo precocemente le caratteristiche di funzione di concentrazione/diluizione, attivazione della vitamina $\mathrm{D}$ ed in seguito di attivatore dell'eritropoiesi, compensa in parte già nelle fasi avanzate dell'insufficienza renale cronica (CKD) delle caratteristiche estremamente interessanti. Queste includono la capacità di eliminare molecole molto tossiche ad alto peso molecolare che, a differenza delle piccole molecole, sono estremamente lesive su numerosi organi ed apparati, quali il sistema cardiovascolare, ma anche direttamente sullo stesso parenchima renale funzionante residuo, a causa delle capacità infiammatorie e pro-ossidanti mirate soprattutto al microcircolo coronarico e renale, all'interstizio ed al tubulo renale. Ricordiamo in particolare le protein bound uremic toxins (PBUT) come l'indoxil-suldato ed il p-cresolo solfato ${ }^{5-7}$ che si legano fortemente all'albumina. Tali molecole non riescono ad essere sufficientemente eliminate da qualsiasi metodica dialitica seppur molto raffinata e/o ad alto potere convettivo. Le emodialisi e i trattamenti ad alta ultrafiltrazione riescono infatti ad eliminare solo la parte protein-free delle PBUT che rappresenta solo il $10 \%$ del loro quantitativo totale., 8 Peraltro la produzione di queste molecole è amplificato

\footnotetext{
'Già Direttore della S.C. Territoriale di Nefrologia e Dialisi ASL di Cagliari, Membro del Gruppo di Studio sul trattamento conservativo della insufficienza renale cronica della Società Italiana di Nefrologia 2S.C. Territoriale di Nefrologia e Dialisi ASL di Cagliari, Cagliari

\section{Corrispondenza:}

Piergiorgio Bolasco, MD, Già Direttore della S.C. Territoriale di Nefrologia e Dialisi ASL di Cagliari, membro del Gruppo di Studio sul trattamento conservativo della insufficienza renale cronica della Società Italiana di Nefrologia, Via Albert Einstein 2I, 09I26 Cagliari, Italy. Email: pg.bolasco@gmail.com
} 
nell'uremia a causa di gravi alterazioni del microbiota intestinale e per le conseguenti alterazioni della permeabilità intestinale. ${ }^{10,11}$ Lo stesso discorso vale per $\mathrm{i}$ fosfati, molecole ambigue a causa della loro distribuzione pluri-compartimentale e per il forte legame del fosfato con il suo raggio idrato che rallenta sensibilmente e con difficoltà l'attraversamento delle membrane dialitiche, anche quelle ad alta porosità. Occorre ricordare che il fosfato è direttamente tossico sul sistema cardiovascolare poiché è ormai ben assodato che liperfosfatemia è indipendentemente correlata con la mortalità cardio-vascolare ma essa stessa, oltre al danno sul microcircolo renale, produce tramite la formazione di microcristalli di idrossiapatite danni irreversibili tubulo-interstiziali renali che accelerano la caduta della FRR. ${ }^{12-14}$

Comunque la FRR tramite una buona diuresi residua produce, anche a valori molto bassi, delle inaspettate ed importantiperdite urinarie di fosfati, grazieall'abbassamento della soglia tubulare dei fosfati. È stato dimostrato in pazienti in dialisi infrequente ma con buona diuresi residua $(\geq 1000 \mathrm{~mL} /$ die) che la FRR residua può produrre un reale balance tra intake fosforico ed output urinario pari a zero grazie anche all'ausilio di una dieta appropriata e ad un congruo uso di chelanti intestinali del fosforo. ${ }^{15-17}$

Alla funzione renale residua occorre aggiungere la prescrizione di una dieta ipoproteica ritagliata sulla FRR e sulla dose dialitica. La dieta ipoproteica risparmia nefroni ed è ormai assodato che rallenta o procrastina l'inizio della terapia sostitutiva poiché preserva la stessa FRR. Da qui si rinforzano le basi per iniziare un trattamento emodialitico incrementale (INC).$^{18}$ Le considerazioni e le formule di Basile et al. ${ }^{19-21}$ definiscono la INC come un trattamento a cui deve essere dedicata una particolare attenzione clinica onde evitare l'utilizzo arbitrario della metodica e raccomandando di non affidare la valutazione di idoneità della FRR esclusivamente ai modelli della cinetica dell'urea.

Infatti, nella nostra esperienza, chi intraprende l'INC nella pratica clinica deve valutare la media della clearance (Kr) della creatinina che sovrastima il VFG con la $\mathrm{Kr}$ dell'urea che lo sottostima, onde calcolare un valore più attendibile della FRR soprattutto quando il VFG diventa $<$ $5 \mathrm{~mL} / \mathrm{min} / 1.73 \mathrm{mq}$. Ma colui che intraprende l'INC deve essere anche consapevole, quando inizia o effettua tale programma che non deve valutare solo questi aspetti; bensì si deve impegnare maggiormente nella sorveglianza clinica e nel valutare $\mathrm{i}$ livelli dei principali analiti ematochimici. Tra questi anche la $\beta 2$-microglobulina molecola che notoriamente nella INC ha livelli plasmatici inferiori rispetto a quelli dei pazienti oligoanurici in dialisi trisettimanale, e questo grazie proprio al valido apporto della FRR. Recentemente, dopo i lavori di Kalantar-Zadek et al. sulla emodialisi bisettimanale e di Bolasco et al ${ }^{22,23}$ sulla dialisi monosettimanale - meglio definita come combined diet dialysis program (CDDP) - si è risvegliato l'interesse di molti studiosi sull'applicazione, in pazienti selezionati, di ritmi dialitici infrequenti. Ciò ha creato confusione sulla esatta definizione nosologica tra dialisi infrequente (INF) rispetto alla INC - non dimenticando che alcuni pazienti in emodialisi trisettimanale che conservano una discreta diuresi possono intraprendere la strada della cosiddetta dialisi decrementale grazie ad un inaspettato aumento del VFG che ha consentito loro di ritornare, seppur per periodi di tempo limitato, alla terapia conservativa in osservazione all'ambulatorio pre-dialisi. Anche le linee guida DOKI 2006 ritengono possibile un approccio alla bisettimanale con una clearance dell'urea $>$ $2-3 \mathrm{~mL} / \mathrm{min} / 1.73 \mathrm{mq} .{ }^{24}$

\section{La dialisi infrequente}

Il tabù fu "spezzato" da una pubblicazione che ha posto i nefrologi di fronte ad una nuova problematica deontologica ${ }^{25}$ : a fronte di un VFG di 3-5 mL/min/1.73 mq è corretto somministrare al paziente un ritmo dialitico trisettimanale? Infatti occorre considerare che la patologia clinica dell'uremia è aggravata dalle patologie dialisidipendenticheproduconoamiloidosi, stress cardiovascolare, microinfiammazione, pro-ossidazione, ecc.

Ben consci della resistenza dei nefrologi americani ad utilizzare ritmi diversi dalla emodialisi trisettimanale si sono sviluppati numerosi lavori di altri studiosi interessati alla INF. Ma la definizione nosologica rimane incorretta, come dimostrato in uno dei primi approcci di Giovannetti et al. nel $1981^{26}$ ove ancora non si conoscevano le potenzialità di applicare ad una emodialisi monosettimanale una dieta ipoproteica molto ristretta. L'errore di definizione continua in Europa ${ }^{27}$ ed oltreoceano: Rhee et al $^{28,29}$ pur descrivendo la potenzialità di una dialisi incrementale preferiscono sviluppare le potenzialità di una dialisi bisettimanale. Numerosi altri autori hanno discusso sulle possibilità di ridurre il ritmo dialitico senza specificare con chiarezza quali ritmi emodialitici settimanali possono essere intrapresi e le scelte ricadono quasi sempre su un ritmo più prudente limitato a due soli trattamenti la settimana. Anche l'inserimento in un ritmo bisettimanale deve essere ben valutato come già fu segnalato da Hanson et al. nel 1999. ${ }^{30}$ Recentemente Tangvoraphonkchai et al. ${ }^{31}$ hanno effettuato una revisione approfondita che riporta tutte le ben note precauzioni quando si sceglie di applicare un ritmo infrequente e/o una variazione del tempo dialisi. Ma di fatto si discute di frequenza non di approccio incrementale e le considerazioni fatte sono ancora basate su speculazioni piuttosto che su casistiche reali. Ancora più confondente la segnalazione di Toth-Manikowski ${ }^{32}$ che immette cinque pazienti in emodialisi domiciliare; per l'Autore l'INC significa comunque aumentare la dose dialitica a fronte ed indipendentemente da un rapido declino di FRR. KalantarZadek et al. ${ }^{33}$ suggeriscono che probabilmente 13.000 pazienti veterani in dialisi avrebbero potuto iniziare con una 
dialisi bisettimanale, definita incrementale, che avrebbe prodotto anche un notevole risparmio sui costi dell' assistenza sanitaria statunitense; tutto questo adducendo una poco convincente valutazione retrospettiva su una migliore qualità di vita rispetto ai centri per pazienti non veterani. Altri autori californiani propongono, definendola INC, per un follow-up di 2 anni, la dialisi bisettimanale ${ }^{34}$; così anche il gruppo spagnolo di Merino et al. ${ }^{35} \mathrm{Da}$ segnalare l'esperienza di Oby et $\mathrm{al}^{36} \mathrm{di}$ confronto tra dialisi convenzionale e dialisi definita incrementale ma ben chiaramente tutti pazienti erano trattati in ritmo bisettimanale. Dopo pochi mesi un'altra osservazione è stata fatta dallo stesso gruppo americano comparando due coorti di pazienti statisticamente troppo sbilanciare sulla sopravvivenza di 50.163 pazienti in dialisi convenzionale versus 434 pazienti in dialisi definita incrementale. Quel che colpisce in questi lavori policentrici americani, a parte l'utilizzo della sola clearance dell'urea per stimare la FRR, è anche la mancanza di qualsiasi riferimento all'applicazione ed utilità di una nutrizione ipoproteica nei pazienti in dialisi INF (definita "twice weekly or less"). Ciononostante i pazienti in INF dimostravano una sopravvivenza, aggiustata per tutte le cause di mortalità, significativamente superiore ai pazienti in emodialisi convenzionale. ${ }^{37}$

\section{La dialisi incrementale}

Le pubblicazioni sulle maggiori riviste europee di nefrologia e dialisi hanno penalizzato l'informazione metodologica e scientifica di quella che è definita la dialisi incrementale in quanto osteggiata dal fatto che lo studio sarebbe dovuto essere condotto, su bracci randomizzati. Purtroppo la FRR non permette eticamente di randomizzare pazienti con VFG così bassi per il rischio di complicanze anche molto serie nel braccio dei pazienti che avrebbero intrapreso il lato "non trattamento". Infatti è obbligatorio ed inevitabile che i pazienti debbano essere necessariamente ed attentamente pre-valutati prima di intraprendere un programma di INC anche se rimane pur sempre un trattamento sostitutivo "bridge" verso il trapianto o verso ritmi dialitici più frequenti durante $\mathrm{i}$ quali si riscontrano minimi segni di uremia, sovraccarico idrico, o complicanze neurologiche di altri apparati e/o organi. Pertanto la INC è definita da un percorso procedurale ben definito.

\section{II percorso}

L'INC inizia di fatto con la valutazione dello status uremico nell' ambulatorio pre-dialisi sinergicamente all'ambulatorio di nefro-nutrizione che allestisce una dieta gradita dal paziente onde assicurare una buona aderenza alimentare ${ }^{23}$ per garantire il mantenimento di un eccellente stato nutrizionale ed una diuresi conservata con un VFG tra i 5 e i $10 \mathrm{~mL} / \mathrm{min} / 1.73 \mathrm{mq}$ eseguendo raccolte urine per calcolare la media tra clearance dell'urea e della creatinina.
All'inizio del programma INC si entra nella fase CDDP. Ogni mese e successivamente ogni tre mesi viene accuratamente calcolata l'urea nitrogen appearance (UNA) secondo la vecchia ma ancora estremamente valida formula di Maroni e Mitch. ${ }^{38}$

Quando si intraprende un programma di dialisi incrementale devono essere presenti requisiti organizzativi e di preparazione degli operatori attivando e mantenendo determinati indispensabili tappe valutative tra cui: valutazione dell'uremia, compliance dietetica, e counseling. Solo così può iniziare il programma monosettimanale (CDDP), con la prescrizione di una dieta iposalina e ipoproteica da $0.6 \mathrm{~g} / \mathrm{kg} / \mathrm{die}$ con aggiunta di aminoacidi essenziali. Il giorno di dialisi il paziente deve avere la soddisfazione di potersi alimentare liberamente. Le principali cause che hanno condotto alla revisione del ritmo monosettimanale sono dovute a cattiva compliance del paziente, incrementi ponderali eccessivi, scompenso cardiaco latente o conclamato, rapido declino della diuresi ${ }^{39}$ e per la presenza di qualsiasi altra causa che produca il men che minimo rischio per il benessere del paziente. Dal 1989 il gruppo di Bolasco $\mathrm{P}$ et $\mathrm{al}^{40}$ ha trattato 126 pazienti senza rilevare alcun caso di scompenso cardiaco o sovraccarico di peso; infatti le più comuni cause di uscita dal programma dei pazienti sono state: riduzione rapida della diuresi e cattiva compliance dietetica. Il vero iniziatore della moderna INC fu il gruppo di Locatelli et al. che abbandonarono il programma IDDP (integrated diet dialysis program) soprattutto per la non aderenza dei pazienti ad una dieta fortemente ipoproteica (very low protein diet, VLPD) con aminoacidi essenziali e loro chetoanaloghi (drop-out $>$ $66 \%) .{ }^{41}$ La nostra esperienza sconsiglia vivamente l'utilizzo di una dieta VLPD nei pazienti in CDDP poiché conduce con altà probabilità alla deplezione proteico calorica (protein energy wasting, PEW). È infine necessario accennare anche quando i pazienti devono abbandonare il programma di dialisi bisettimanale, ovvero quando si realizzano le stesse cause di abbandono di un programma CDDP.

Infatti utilizzando un ritmo dialitico bisettimanale occorre delineare quando i pazienti devono abbandonarlo: le cause sono le stesse dell'abbandono dalla CDDP; occorre valutare strettamente e clinicamente i pazienti poiché essenzialmente ciò accade per: la riduzione della diuresi (poiché questa è spesso subdola e inavvertita), incrementi interdialitici eccessivi ( $>3-5 \%$ del peso secco), stato in cui effettuando la media tra $\mathrm{Kr}$ urea e $\mathrm{Kr}$ creatinina il VFG è $\leq 1.5 / \mathrm{mL} / \mathrm{min} / 1.73 \mathrm{mq}$, ed il paziente deve entrare in ritmo trisettimanale in linea con quanto espresso dalle linee KDOQI $2006 .{ }^{24}$ La differenza tra il nostro programma e gli altri segnalati nella letteratura è che anche in emodialisi bisettimanale occorre raccomandare al paziente una attenta osservanza di una dieta "ipoproteica" tra $0.9-1 \mathrm{~g} / \mathrm{Kkg} / \mathrm{die}$. Nella nostra casistica in CDDP e bisettimanale abbiamo notato assai raramente che il paziente superi la soglia $>5 \mathrm{mg} / \mathrm{dL}$ di fosforemia. 


\section{Conclusioni}

Per il nefrologo, l'infermiere di nefrologia e dialisi e soprattutto per il paziente è fondamentale la distinzione nosologica tra dialisi infrequente ed incrementale.

La dialisi infrequente è una scelta che privilegia e applica dei programmi di trattamento meno frequenti ed è ben differente dalla dialisi incrementale e questo non solo etimologicamente. Intraprendere la dialisi incrementale, infatti, significa aaviare un percorso ben preciso, che veda applicato un determinato protocollo e che necessita l'applicazione di un itinerario progressivo operato da un team con una precisa organizzazione a favore del mantenimento della FRR del paziente la quale è di grande interesse soprattutto nelle more di evitare l'overdose dialitica soprattutto per i pazienti inseriti in un programma transplantologico.

\section{Dichiarazione di assenza di conflitto di interessi}

Gli Autori dichiarano di non aver alcun conflitto di interesse. Non è stata esercitata alcuna influenza intellettuale, commerciale sulla stesura e conclusione del lavoro da parte di Aziende o Enti privati.

\section{Bibliografia}

1. Chandna SM e Farrington K. Residual renal function: considerations on its importance and preservation in dialysis patients. Semin Dial 2004; 17: 196-201.

2. Vilar E, Wellsted D, Chandna SM, et al. Residual renal function improves outcome in incremental haemodialysis despite reduced dialysis dose. Nephrol Dial Transplant 2009; 24: 2502-2510.

3. Viglino G, Neri L e Barbieri S. Incremental peritoneal dialysis: effects on the choice of dialysis modality, residual renal function and adequacy. Kidney Int Suppl 2008;108: S52-S55.

4. Neri L, Viglino G, Cappelletti A, et al. Incremental dialysis with automated peritoneal dialysis. Adv Perit Dial 2003; 19: 93-96.

5. Deltombe O, Van Biesen W, Glorieux G, et al. Exploring Protein Binding of Uremic Toxins in Patients with Different Stages of Chronic Kidney Disease and during Hemodialysis. Toxins (Basel) 2015; 7: 393-396.

6. Wu IW, Hsu KH, Lee CC, et al. P-cresyl sulphate and indoxyl sulphate predict progression of chronic kidney disease. Nephrol Dial Transplant 2011; March 26: 938947.

7. Vanholder R, Schepers E, Pletinck A, et al. The uremic toxicity of indoxyl sulfate and p-cresyl sulfate: a systematic review. J Am Soc Nephrol 2014; 25: 1897-1907.

8. Glorieux G e Vanholder R. New uremic toxins - which solutes should be removed? Contrib Nephrol 2011;168: 117-28.

9. Neirynck N, Glorieux G, Schepers E, et al. Review of protein-bound toxins, possibility for blood purification therapy. Blood Purif 2013; 35 Suppl 1: 45-50.
10. Borges NA, Barros AF, Nakao LS, et al. Protein-Bound Uremic Toxins from Gut Microbiota and Inflammatory Markers in Chronic Kidney Disease. J Ren Nutr 2016; 26: 396-400.

11. Gryp T, Vanholder R, Vaneechoutte M, et al. p-Cresyl Sulfate. Toxins (Basel) 2017; 9: 52.

12. Neves KR, Graciolli FG, dos Reis LM, et al. Adverse effects of hyperphosphatemia on myocardial hypertrophy, renal function, and bone in rats with renal failure. Kidney Int 2004; 66: 2237-2244.

13. Lau K. Phosphate excess and progressive renal failure: the precipitation-calcification hypothesis. Kidney Int 1989; 36: 918-937.

14. Zoccali C, Ruggenenti P, Perna A, et al. Phosphate may promote $\mathrm{CKD}$ progression and attenuate renoprotective effect of ACE inhibition. J Am Soc Nephrol 2011; 22: 19231930.

15. Bolasco P, Murtas S, Caria S, et al. The Power of Phosphaturia in the Infrequent Hemodialysis. G Ital Nefrol 2017; 34(1).

16. Bolasco P e Murtas S. Clinical benefits of phosphate control in progression of end stage renal disease. Panminerva Med 2017; 59: 133-138.

17. Bolasco P, Murtas S, Galfré A, et al. Phosphate Nutritional Intake Control between Patient Undergoing Conventional Thrice Weekly and Infrequent Hemodialysis. International Journal of Clinical Nutrition 2017; 5: 18-23.

18. Locatelli F, Del Vecchio L e Aicardi V. Nutritional Issues with Incremental Dialysis: The Role of Low-Protein Diets. Semin Dial 2017; 30: 246-250.

19. Casino FG e Basile C. The variable target model: a paradigm shift in the incremental haemodialysis prescription. Nephrol Dial Transplant 2017; 32: 182-190.

20. Casino FG e Basile C. How to set the stage for a full-fledged clinical trial testing 'incremental haemodialysis. Nephrol Dial Transplant. Epub ahead of print 21 July 2017. DOI: 10.1093/ndt/gfx225

21. Basile C, Casino FG e Kalantar-Zadeh K. Is incremental hemodialysis ready to return on the scene? From empiricism to kinetic modelling. J Nephrol 2017; 30: 521-529.

22. Kalantar-Zadeh K, Unruh M, Zager PG, et al. Twice-weekly and incremental hemodialysis treatment for initiation of kidney replacement therapy. Am J Kidney Dis 2014; 64: 181-186.

23. Bolasco P, Caria S, Egidi MF, et al. Incremental approach to hemodialysis: twice a week, or once weekly hemodialysis combined with low-protein low-phosphorus diet? G Ital Nefrol 2015; 32(6).

24. National Kidney Foundation. KDOQI Clinical Practice Guideline for Hemodialysis Adequacy: 2015 update. Am J Kidney Dis 2015; 66: 884-930.

25. Kalantar-Zadeh K e Casino FG. Let us give twice-weekly hemodialysis a chance: revisiting the taboo. Nephrol Dial Transplant 2014; 29: 1618-1620.

26. Barsotti G, Guiducci A, Ciardella F, et al. Effects on renal function of a low-nitrogen diet supplemented with essential amino acids and ketoanalogues and of hemodialysis and free protein supply in patients with chronic renal failure. Nephron 1981; 27:113-117. 
27. Wong J, Vilar E, Davenport A e Farrington K. Incremental haemodialysis. Nephrol Dial Transplant. 2015 Oct;30(10):

28. Rhee CM, Ghahremani-Ghajar M, Obi Y, et al. Incremental and infrequent hemodialysis: a new paradigm for both dialysis initiation and conservative management. Panminerva Med 2017; 59: 188-196.

29. Rhee CM, Unruh M, Chen J, et al. Infrequent dialysis: a new paradigm for hemodialysis initiation. Semin Dial 2013; 26: $720-727$.

30. Hanson JA, Hulbert-Shearon TE, Ojo AO, et al. Prescription of twice-weekly hemodialysis in the USA. Am J Nephrol 1999; 19: 625.

31. Tangvoraphonkchai $\mathrm{K}$ e Davenport A. Increasing Haemodialytic Clearances as Residual Renal Function Declines: An Incremental Approach. Blood Purif 2017; 44: 217-226.

32. Toth-Manikowski SM, Mullangi S, Seungyoung Hwang $\mathrm{S}$, et al. Incremental short daily home hemodialysis: a case series. BMC Nephrol 2017; 18: 216.

33. Kalantar-Zadeh K, Crowley ST, Beddhu S, et al. Renal Replacement Therapy and Incremental Hemodialysis for Veterans with Advanced Chronic Kidney Disease. Semin Dial 2017; 30: 251-261.

34. Ghahremani-Ghajar M, Rojas-Bautista V, Lau WL, et al. Incremental Hemodialysis: The University of California Irvine Experience. Semin Dial 2017; 30: 262-269.
35. Merino JL, Domínguez P, Bueno B, et al. Application of a pattern of incremental haemodialysis, based on residual renal function, when starting renal replacement therapy. Nefrologia 2017; 37: 39-46.

36. Obi Y, Streja E, Rhee CM, et al. Incremental Hemodialysis, Residual Kidney Function, and Mortality Risk in Incident Dialysis Patients: A Cohort Study. Am J Kidney Dis 2016; 68: 256-265.

37. Mathew A, Obi Y, Rhee CM, et al. Treatment frequency and mortality among incident hemodialysis patients in the United States comparing incremental with standard and more frequent dialysis. Kidney Int 2016; 90: 1071-1079.

38. Maroni BJ, Steinman TI e Mitch WE. A method for estimating nitrogen intake of patients with chronic renal failure. Kidney Int 1985; 27: 8-65.

39. Libetta C, Esposito P e Dal Canton A. Once-weekly hemodialysis: a single-center experience. Am J Kidney Dis 2015; 65: 343.

40. Bolasco P, Cupisti A, Locatelli F, et al. Dietary Management of Incremental Transition to Dialysis Therapy: OnceWeekly Hemodialysis Combined With Low-Protein Diet. $J$ Ren Nutr 2016; 26: 352-359.

41. Locatelli F, Andrulli S, Pontoriero G, et al. Integrated diet and dialysis programme. Nephrol Dial Transplant 1998; 13 (Suppl 6): 132-138. 